\title{
Resonant Polarization Spectroscopy for Hot X-ray
}

\section{Plasmas}

\author{
Guo-Xin Chen \\ Harvard-Smithsonian Center for Astrophysics, 60 Garden Street, Cambridge, MA 02138, USA
}

\begin{abstract}
X-ray line polarization spectroscopy is a method of choice for probing hot plasma conditions. The precise roles of resonant structures in this method have not been realized and fully understood. With a sophisticated relativistic close coupling Dirac R-matrix calculation of polarized radiation of the quadrupole magnetic M2 line at $2.717 \AA$ in $\mathrm{Ba}^{46+}$, we revealed the nature of resonant structures in x-ray line polarization spectroscopy. We found that signatures with a heavy resonance forest imprinting on polarization may be used for a sensitive new spectroscopic method. The resonant polarization spectrum was used to determine or constrain the directional beam electron distribution of the laboratory Ba plasma. Our results provide a start of resonant polarization spectroscopy as a method for diagnostics of laboratory, fusion and astrophysical plasma source conditions.
\end{abstract}

Key words: Relativistic, resonance, polarization, spectroscopy, plasmas.

\section{Introduction}

Polarization spectroscopy [1] of radiation or fluorescence is an important method of choice for probing structural, dynamical, and physical properties in a broad spectrum of areas ranging from hot laser-produced plasmas [2, 3], inertial confinement fusion [4, 5], and magnetic confinement fusion plasmas [6] to small-scale laboratory [7-9] and cosmic-scale astrophysical plasmas $[10,11]$ to biophysics [12]. The degree of polarization of the emitted radiation, which is directly connected to the anisotropy in the velocity space of an ensemble of excited atomic particles inside a medium, may also be used for remotely sensing the structural features of ionized medium from spectra observed in laser-plasmas interaction and the interaction of ion-beams and surface in microlithography [13-15].

Polarization of $\mathrm{x}$-ray line radiation can be generated when an alignment of MS (magnetic sublevels) of an atomic target is created by directional electron beams or magnetic fields $[1,16]$. Alignment is a consequence

Corresponding author: Guo-Xin Chen, Ph.D., physicist, research fields: relativistic atomic physics and X-ray astrophysics. when the MS population of the upper fine-structure atomic level for a given x-ray line is unequal. In non-thermal hot plasmas, $\mathrm{x}$-ray line polarization spectroscopy $[2,16-18]$ is an essential tool (a) for probing physical conditions, such as electron density and temperature distributions, ion charge state and abundance distributions, and electron angular and anisotropic velocity distributions [3, 19-23]; and (b) for studying plasma energy and radiation transport [3, $18,20]$. X-ray line polarization spectroscopy has been applied to determine efficiently the non-thermal EDF (electron distribution functions) of (i) high energy density laser-produced plasmas [2, 3, 13], (ii) inertial confinement fusion plasmas [5, 24-27], and (iii) low density magnetic confinement fusion plasmas [6, 28-30]. X-ray line polarization spectroscopy may also be used to study quantitatively x-ray formation physics of highly charged complex ions in laboratory plasmas [2, 14, 31, 32].

In $\mathrm{x}$-ray astrophysics, polarization analysis of observed x-ray line spectra (along with continuum spectra) opens a new window to infer the geometry and angular distribution of line emitting sources [10, $11,33]$. X-ray line polarization spectroscopy may be 
applied to reveal the nature of violent and exotic sources in space, such as solar flares [34-36], supernova blast waves [34, 37], clusters of galaxies and gamma-ray burst afterglows [11, 38-40]. The observation of $\mathrm{x}$-ray polarization provides an insightful vision for and a unique test of the new physics in these fascinating $\mathrm{x}$-ray emitting sources. The diagnostic of the radiation emission from hot plasmas in solar flaring regions is a key to the ultimate aim to provide predictions or forecasts of solar activity and "space weather" [41].

The resonance contributions to both the electron excitation cross sections and components of the polarized radiative emission have been systematically treated in a number of previous works (see e.g. [1, 3]). However, the precise roles of resonant structures in $\mathrm{x}$-ray line polarization spectroscopy have not been realized and fully understood. In this work, we reveal the nature of resonant structures in $x$-ray line polarization spectroscopy, and advance a diagnostic method RPS (resonant polarization spectroscopy), rearming the present hot plasma diagnostic tools. While resonance effects were considered in previous models, $\mathrm{x}$-ray line polarization spectrum with resonant structures identified has never been used before as a plasma spectroscopy tool. The power of our RPS method relies critically on the sophisticated relativistic calculation of resonant structures in the polarization spectrum and the correct identification of these resonant structures.

\section{Method and Calculation}

Our new RPS method is demonstrated here in two steps: (1) We first report fully relativistic quantum mechanical calculation of polarized radiation of the quadrupole magnetic line (M2) at 4,563 eV or $2.717 \AA$ $\left(2 p^{5} 3 s^{3} p_{2}^{0} \rightarrow 2 p^{6}{ }^{1} S_{0}\right)$ in highly charged Ne-like ion $\mathrm{Ba}^{46+}$. The calculation of $\mathrm{x}$-ray line polarization spectroscopy was carried out by the relativistic close coupling Dirac R-matrix method we developed in Ref. [16]. We took into account the effects of interacting
Rydberg series of resonances (with the inclusion of radiation damping to resonances) on MS EIE (electron impact excitation) cross sections, and the effects of cascade on M2 MS line intensities from many high-lying MS. We found that signatures, with a heavy resonance forest imprinting on $x$-ray line polarization spectrum, may be a sensitive diagnostic tool. (2) The resonant polarization spectrum was then used to determine or constrain the plasma characteristics, such as the directional beam electron $\mathrm{EDF}$, of the laboratory Ba plasma developed on an EBIT (electron beam ion trap) [42].

When the intensities of the two polarization-specific components of the x-ray line are different, the presence of anisotropy in plasmas may be characterized by polarization degree $P(E)$ as a function of electron energy $E$, defined by $P(E)=\left[I_{11}(E)\right.$ $\left.-I_{\perp}(E)\right] /\left[I_{11}(E)+I_{\perp}(E)\right]$, where $I_{\mathrm{ll}}(E)$ and $I_{\perp}(E)$ are the polarized photon intensities with electric field vectors parallel and perpendicular to the plane defined by the electron-beam direction (the axis of symmetry) and the observation line of sight. In the laboratory EBIT plasmas, $\mathrm{x}$-ray emission is often observed at $90^{\circ}$ to the electron beam [42]. The detailed polarization is then calculated from MS EIE cross sections $\sigma_{2}$ for $M_{f}=2$ and $\sigma_{1}$ for $M_{f}=1$ ( $M_{f}$ is the magnetic quantum number of the final or upper level in the MS transition) [17], $P(E)=\left[\sigma_{2}(E)-\sigma_{1}(E)\right] /\left[\sigma_{2}(E)+\sigma_{1}(E)\right]$.

The relativistic close coupling DRM (Dirac R-matrix) method we have developed in Ref. [16] was employed to calculate $\mathrm{x}$-ray line polarization. Both channel coupling effects and interacting series of Rydberg resonances were readily included by our DRM method. We included target states up to the principal quantum number $n=5$ in the relativistic atomic structure calculation of $\mathrm{Ba}^{46+}[17]$.

Large resonance effects and complicated resonant structures in EIE collision strengths of M2 MS were demonstrated in Fig. 1. The strength of resonances may dominate over the non-resonant background of M2 MS collision strengths. The combined constructive 


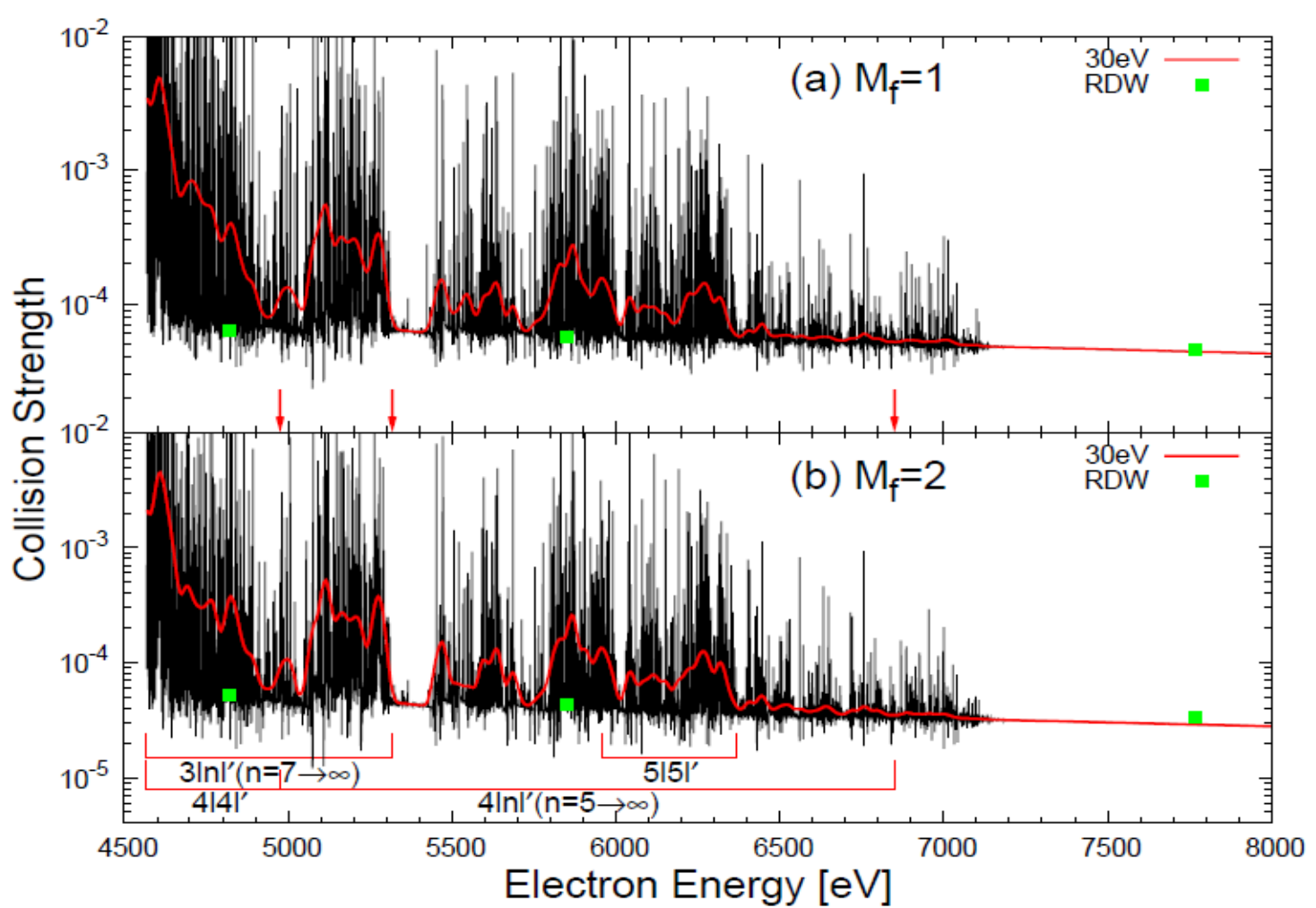

Fig. 1 Detailed MS EIE collision strengths $\Omega=w_{i} k_{i}{ }^{2} \sigma$ against the incident electron energy $E$ for M2 transition with magnetic quantum number $M_{f}=1$ (a) and $M_{f}=2(\mathrm{~b})$, where $w_{i}$ is the statistical weight of the initial state. The red thick curve is the averaged collision strengths $\bar{\Omega}$ when the detailed collision strengths $\Omega$ are convolved by a Gaussian EDF with a beam width of $W=30 \mathrm{eV}$. Filled squares: previous RDW (relativistic distorted-wave) calculations [43].

and destructive effects of interacting Rydberg series of resonances may significantly change the non-resonant background collision strengths. The consequence of all these effects is reflected in the polarization calculation given below. In Fig. 1, we were also able to identify the major Rydberg resonance series in MS atomic collision processes. The left red arrow at $E=$ 4,975.6 eV marks the threshold for $4 \ell 4 \ell^{\prime}$ resonances; the middle red arrow at $E=5,318.3 \mathrm{eV}$ and the right red arrow at $E=6,849.8 \mathrm{eV}$ mark respectively the Rydberg resonance series of $3 \ln \ell^{\prime}(n=7-\infty)$ and $4 \ln \ell^{\prime}$ $(n=5-\infty)$ in M2 MS collision strengths. Here $\ell$ is the orbital quantum number and $n \ell n^{\prime} \ell^{\prime}$ denote doubly excited resonance states. The interacting Rydberg resonance series of $5 \ell n \ell^{\prime}(n=5-\infty)$ appear at higher energy of $E>5,959.3 \mathrm{eV}$. These strong Rydberg series of resonances of $4 \ell 4 \ell^{\prime}$ and $3 \ell n \ell^{\prime}(n=7-\infty)$ shown in Fig. 1 are most important in demonstrating the RPS method detailed below.

M2 is a forbidden transition, unlike the electric dipole transition shown in Ref. [16], so many interacting Rydberg series of resonances are strong for an extended range of electron energy. This is the main reason to use M2 polarization to demonstrate our RPS method. However, cascade effects on M2 polarization from many high-lying MS need to be taken into account at high $E$ region, so polarization of a large number of high-lying MS transitions must first be explicitly calculated. An extensive MS CRM (collisional-radiative model) with MS collision and radiative rates was then constructed to calculate the cascade effects. This MS CRM is similar to the FS (fine-structure) CRM used in Ref. [44], except that the FS collision rates in Ref. [44] were replaced by the MS collision rates, and the FS Einstein radiative transition coefficients were replaced by MS radiative 
transition coefficients.

\section{Results}

Fig. 2 shows the effects of cascade for M2 polarization. Two previous RDW (relativistic distorted-wave) results $[42,43]$ are also given for comparisons. For $E$ below the $n=3$ target threshold, the cascade effects significantly reduce the amplitude of oscillatory features, which are due to resonances in MS collision strengths, in M2 polarization. But the effects on the non-oscillation background of $\mathrm{M} 2$ polarization are less significant. For $E$ above $n=3$ target threshold, cascade has significant effects on both the oscillatory features and the non-oscillation background of M2 polarization. The non-oscillation background is raised by about a factor of two due to cascade effects. However, it was shown that the M2 polarization increases by about a factor of four due to cascade effects from nonzero angular momentum levels [42]. There is about a factor of two difference between the present DRM0 calculation without cascade and the earlier RDW0 calculation [42]. Although there is some amount of agreement for the backgrounds of M2 polarization among the present calculation (DRM1 with cascade effects), the RDW1 calculation in Ref. [42] (with cascade effects), and the RDW2 calculation in Ref. [43] (without cascade effects), such agreement is fortuitous, as detailed above and shown in Fig. 2. Some rather limited resonance effects on M2 polarization were also calculated by the RDW method in Ref. [43]. However, in low energy region, this RDW calculation missed the prominent resonances of $4 \ell 4 \ell^{\prime}$ and $3 \ell n \ell^{\prime}(n=7-\infty)$, which in turn are most important for the demonstration of the new RPS method advanced in this work.

The M2 polarization was further calculated and shown with the convolution of Gaussian beam width at $W=10 \mathrm{eV}$ and $30 \mathrm{eV}$ in Fig. 3 and at $W=5 \mathrm{eV}$ in Fig. 4. We found that signatures with a heavy resonance forest shown in Fig. 1 are imprinted on the M2 x-ray polarization. The oscillation features in M2

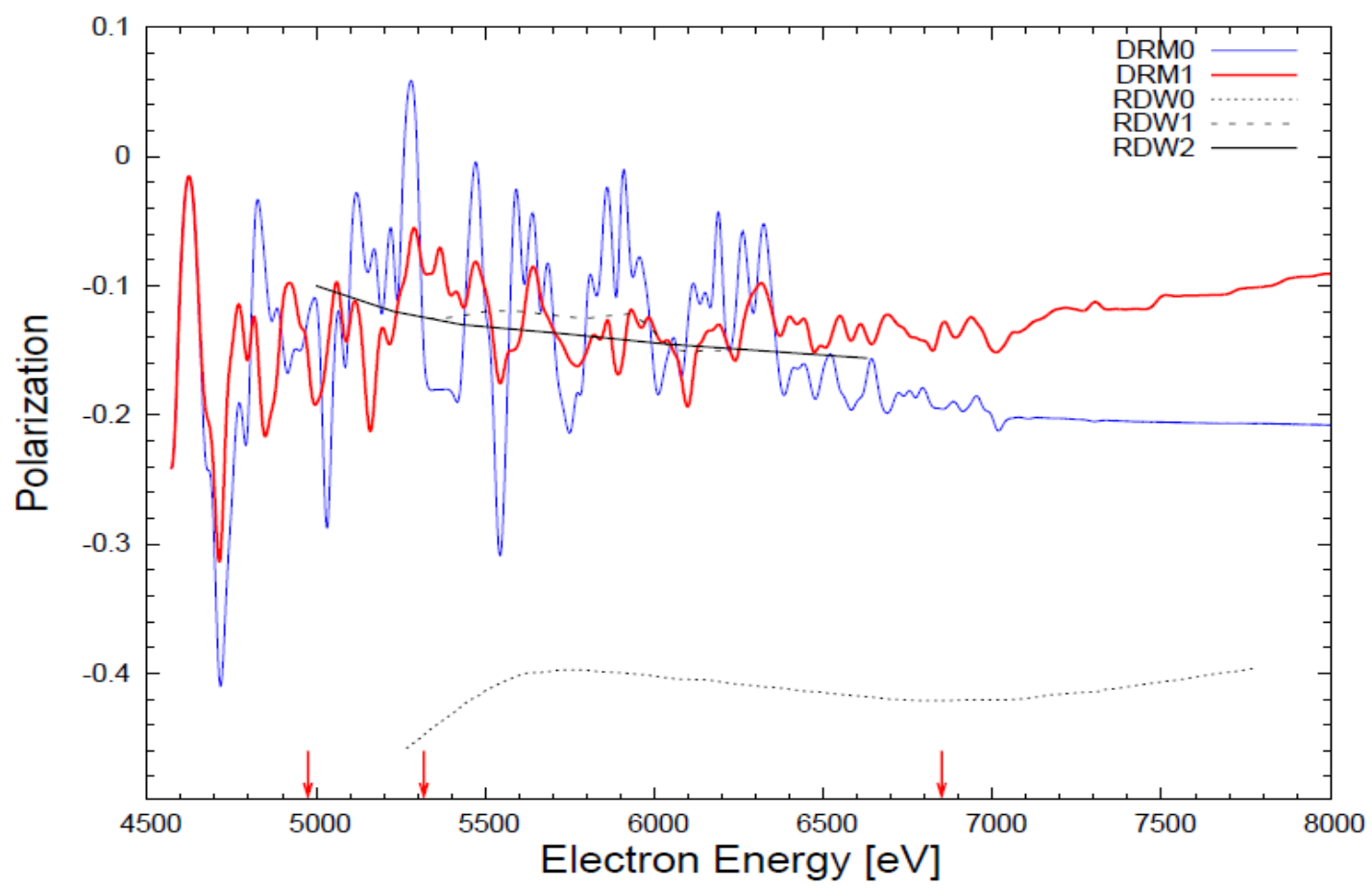

Fig. 2 Cascade effects in M2 polarization of $\mathrm{Ba}^{46+}$. DRM0 (blue thin curve) and DRM1 (Red thick curve): averaged polarization for $W=30 \mathrm{eV}$ without and with cascade, respectively; dotted curve: RDW0 results from Ref. [42] without cascade from nonzero angular momentum levels; dashed curve: RDW1 results from Ref. [42] with cascade; solid black curve: RDW2 results from Ref. [43] without cascade. The three red arrows mark resonance thresholds as explained in Fig. 1a. 


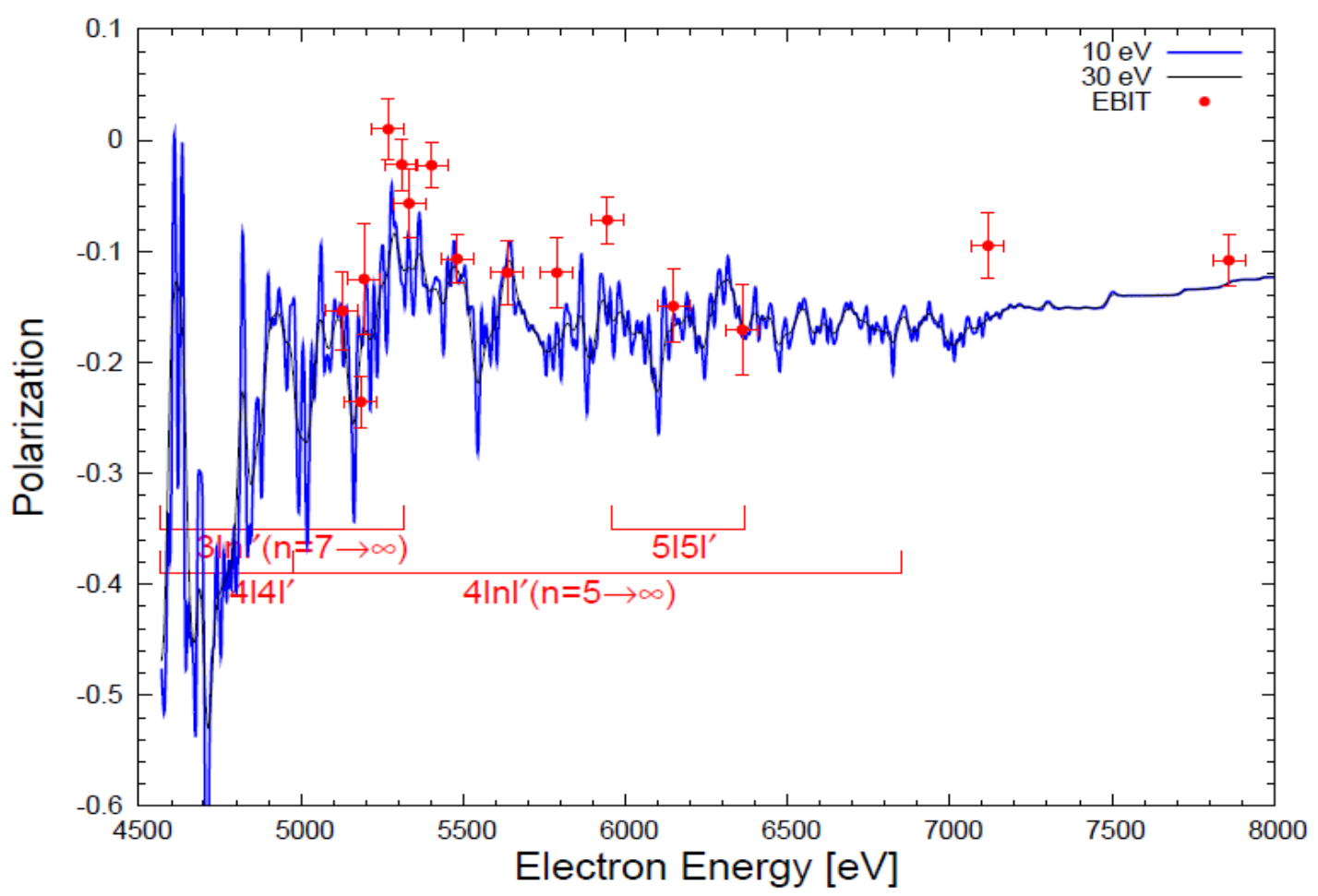

Fig. 3 Averaged M2 polarization of $\mathrm{Ba}^{46+}$ from the convolution of detailed polarization with Gaussian EDF at electron beam width $W=10 \mathrm{eV}$ (blue thick curve) and $W=30 \mathrm{eV}$ (black thin curve). The filled dots with error bars are polarization observed in the EBIT Ba plasma [42].

polarization are caused by the Rydberg resonance series of $3 \ell n \ell^{\prime}(n=7-\infty)$ and $4 \ell 4 \ell^{\prime}$ resonances in $\mathrm{M} 2$ MS collision strengths for $E<5,000 \mathrm{eV}$, and by the Rydberg resonance series of $4 \ell n \ell^{\prime},(n=5-\infty)$ and $5 \ell n \ell^{\prime}$, $(n=5-\infty)$ for $E>5,000 \mathrm{eV}$, all of which are identified and explained in Fig. 1. In Figs. 3 and 4, it should be noted that our DRM calculation of the M2 polarization spectrum was normalized to the E1 line at $4,568 \mathrm{eV}$.

Next, our theoretical polarization spectrum was further applied to determine or constrain the directional beam EDF of the laboratory EBIT plasmas $[8,32,42]$. EBIT plasmas is an ideal test bed to explore $\mathrm{x}$-ray formation physics and atomic physics of highly charged ions, important for studying hot plasmas in laser-produced, controlled fusion and astrophysical sources. EBIT may also be an ideal extraction system of ion beam sources for studying ion-surface interaction in microlithography [14, 15]. Similar to astrophysical plasmas, such as the matter of sparse interstellar gases, $\mathrm{x}$-rays emitted from highly charged ions in EBIT plasmas result from the interaction of a beam of high-energy electrons with a cloud of ions. The x-ray lines emitted from excited MS are polarized because of the anisotropy of EBIT beam's EDF. The study of polarization is thus key to the physical interpretation and analysis of $\mathrm{x}$-ray radiation in EBIT plasmas. It is now known that a new area of EBIT science emerges on the study of the EBIT plasma as an exotic source and for the understanding of fine details of the EBIT plasma confinement, such as the dynamics of electron beam and ion cloud in non-neutral EBIT plasmas; the plasma heating, cooling, electromagnetic interaction or collisions in transient or equilibrium EBIT systems $[31,45-48]$.

The energy $E$ and the spread $W$ of the electron beam are two key parameters that characterized an EBIT plasma. The power of our RPS method is shown below by the application of the method to determine or infer $E$ and $W$. Because of the lacking of a sophisticated calculation for and a correct identification 


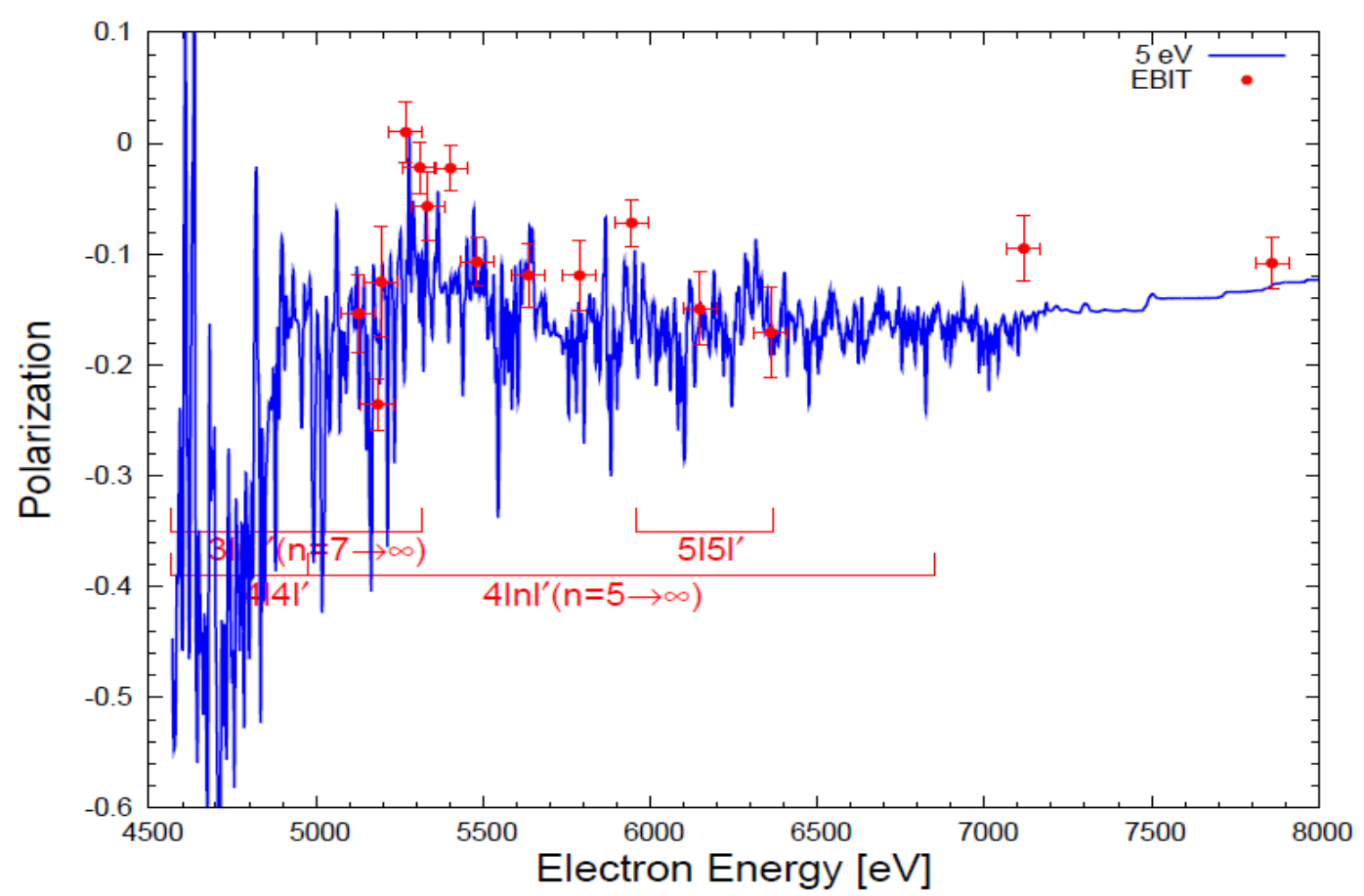

Fig. 4 Averaged $\mathrm{M} 2$ polarization of $\mathrm{Ba}^{46+}$ from the convolution of detailed polarization with a Gaussian EDF at electron beam width $W=5 \mathrm{eV}$ (blue curve). The filled dots with error bars are polarization observed in the EBIT Ba plasma [42].

of resonant structures in previous models [42, 43], it is impossible for previous works to infer both the parameters. Although the energy shift due to space charge (shifts with respect to the drift tube electrode voltage of EBIT) can be easily determined, the partial neutralization of the trapped positive ions is hard to specify [31, 42]. The position of electron beam energy is therefore difficult to specify accurately [31, 42]. It is also important to know precisely the EBIT beam spread $W$ for the study of $x$-ray formation physics and spectral modeling of EBIT plasmas [46]. The EBIT beam spread $W$ is largely due to the radial variation of space charge shift through the electron beam. The precise beam spread $W$ is another parameter that is hard to specify for EBIT plasmas [31, 42].

The EBIT polarization data were measured simultaneously with two identical bent crystal $\mathrm{x}$-ray spectrometers [42]. In Figs. 3 and 4, we apply our M2 theory polarization to the determination or constraint of the beam width $W$ and the beam energy shift due to space charge with neutralization correction. Compared with the case of $W=30 \mathrm{eV}$, our polarization with $W$ close to $10 \mathrm{eV}$ is shown to be much closer to the observed polarization under the EBIT Ba plasma conditions [42]. Hence, we infer that the beam width in this EBIT Ba plasma is below $W=10 \mathrm{eV}$ and is well below $30 \mathrm{eV}$. We note that some similar small beam widths of about $10 \mathrm{eV}$ have been observed in some other EBIT plasmas [49, 50].

From Figs. 3 and 4, an overall beam energy correction in the EBIT Ba plasma, due to space charge and its neutralization, was determined to be about 100 $\mathrm{eV}$ [42]. The uncertainty of the beam energy reported in Ref. [42] is $\pm 50 \mathrm{eV}$.

\section{Summary}

In summary, we have carried out a sophisticated and fully relativistic quantum mechanical calculation, with the inclusion of resonant structures and cascade effects, of the M2 $\mathrm{x}$-ray line polarization in $\mathrm{Ba}^{46+}$. Our resonant polarization spectrum was applied to the diagnostics of the laboratory EBIT Ba plasma. The 
beam energy shift due to space charge and the beam spread, which are two key EBIT plasma parameters, were determined or constrained by our new RPS method. We anticipate that our work will provide a start of resonant polarization spectroscopy as a general method for insightful diagnostics of non-thermal plasma characteristics. Our method may be used in the precise determination of anisotropic EDF in a wider range (other than $\mathrm{x}$-ray band) of laboratory, fusion and space plasmas important for further study of plasma energy and radiation transport.

This work was performed in part under the auspices of the U.S. Department of Energy grant DE-NA0001802 and the Chandra X-ray Observatory theory grant TM3-14002X.

\section{References}

[1] Fujimoto, T., and Iwamae, A. 2008. Plasma Polarization Spectroscopy. Berlin: Springer-Verlag.

[2] Kieffer, J. C. et al. 1992. Phys. Rev. Lett. 68: 480; ibid., 1993. 48: 4648.

[3] Kawamura, T. et al. 2007. Phys. Rev. Lett. 99: 115003.

[4] Ivanov, V. V. et al. 2009. Phys. Rev. E 79: 056404.

[5] Ouart, N. D. et al. 2014. Phys. Plasmas 21: 031207.

[6] Fujimoto, T. et al. 1996. Phys. Rev. E 54: R2240.

[7] Levine, M. A., Marrs, R. E., Henderson, J. R., Knapp, D. A., and Schneider, M. B. 1988. Phys. Scripta T22: 157.

[8] Gillaspy, J. 2012. Am. Inst. Phys. Conf. Proc. 1438: 97.

[9] Bernitt, S. et al. Nature 492: 225.

[10] Costa, E. et al. 2001. Nature 411: 662.

[11] Dorodnitsyn, A., and Kallman, T. 2010. Astrophys. J. 711: L112.

[12] Lakowitz, J. R. 2006. Principles of Fluorescence Spectroscopy. 3rd ed. Berlin: Springer-Verlag; Owicki, J. C. 2000. J. Biomol. Screen. 5: 297.

[13] Woolsey, N. C. et al. 2011. High Energy Dens. Phys. 7: 105.

[14] Gillaspy, J. D. 2001. J. Phys. B: At. Mol. Opt. Phys. 19: R93.

[15] Berggren, K. K. et al. 1995. Science 269:1255.

[16] Chen, G. X., Kirby, K., Nancy, N. S., Lin, T., and Silver, E. 2009. Phys. Rev. A 79: 062715 (2009); Chen, G. X. 2011. Phys. Rev. A 84: 012705, and references therein.

[17] Inal, M. K., and Dubau, J. 1987. J. Phys. B: At. Mol. Opt. Phys. 20: 4221.

[18] Hakel, P., Mancini, R. C., Abdallah, J., Sherrill, M. E., and Zhang, H. L. 2009. J. Phys. B: At. Mol. Opt. Phys. 42: 085701 .
[19] Dubau, J., Inal, M. K., and Urnov, A. M. 1996. Phys. Scripta T65: 179.

[20] Inubushi, Y. et al. 2006. J. Quant. Spectrosc. Radiat. Transfer 99: 305; ibid. 101: 191.

[21] Booth, N. et al. 2011. Nucl. Instr. Meth. Phys. Res. A653: 137.

[22] Bedrane, Z., Inal, M. K., and Fritzsche, S. 2009. J. Phys. B: At. Mol. Opt. Phys. 42: 055701.

[23] Smith, R. K., Chen, G. X., Kirby, K., and Nancy, N. S. 2009. Astrophys. J. 700: 679.

[24] Hammel, B. A. et al. 1993. Phys. Rev. Lett. 70: 1263.

[25] Kantsyrev, V. L., Fedin, D. A., Shlyaptseva, A. S., Hansen, S., Chamberlain, D., and Ouart, N. 2003. Phys. Plasmas 10: 2519.

[26] Shlyaptseva, A. S. et al. 2001. Rev. Sci. Instrum. 72: 1241.

[27] Safronova, A. S. et al. 2006. IEEE Transactions on Plasma Science 34: 2256.

[28] Weinheimer, J., Ahmad, I., Herzog, O., Kunze, H. J., Bertschinger, G., Biel, W., Borchert, G., and Bitter, M. 2001. Rev. Sci. Instrum. 72: 2566.

[29] Bertschinger, G., Biel, W., Herzog, O., Weinheimer, J., Kunze, H. J., and Bitter, M. 1999. Phys. Scripta T83: 132.

[30] Coda, S. 2008. Rev. Sci. Instrum. 79: 10F501.

[31] Takacs, E. et al. 2003. Nucl. Instr. Meth. Phys. Res. B205: 144.

[32] Brown, G. V., and Beiersdorfer, P. 2012. Phys. Rev. Lett. 108: 139302, and references therein. Brown, G. V. et al. 2006. Phys. Rev. Lett. 96: 253201.

[33] Henoux, J. C., and Vogt, E. 1998. Phys. Scripta T78: 60.

[34] Laming, J. M. 1990. Astrophys. J. 362: 219; ibid. 357: 275.

[35] Haug, E. 1979. Sol. Phys. 61: 129; ibid. 1981. 71: 77.

[36] Vocks, C., Mann, G., and Rausche, G. 2008. Astro. Astrophys. 480: 527.

[37] Weisskopf, M. C., Silver, E. H., Kestenbaum, H. L., Long, K. S., and Novick, R. 1978. Astrophys. J. 220: L117.

[38] Santos-Lleo, M., Schartel, N., Tananbaum, H., Tucker, W., and Weisskopf, M. C. 2009. Nature 462: 997; Reeves, J. N. et al. 2002. Nature 416: 512.

[39] Sazonov, S. Y., Churazov, E. M., and Sunyaev, R. A. 2002. Mon. Not. R. Astr. Soc. 333: 191.

[40] Zhuravleva, I. V., Churazov, E. M., Sazonov, S. Y., Sunyaev, R. A., Forman, W., and Dolag, K. 2010. Mon. Not. R. Astr. Soc. 403: 129.

[41] Hapgood, M. 2012. Nature 484: 311; Hasegawa, H. et al. 2004. Nature 430: 755.

[42] Takacs, E. et al. 1996. Phys. Rev. A 54: 259.

[43] Jiang, J., Dong, C. Z., Xie, L. Y., and Wang, J. G. 2008. Phys. Rev. A 78: 022709.

[44] Chen, G. X. 2008. Mon. Not. R. Astr. Soc. 386: L62. 
[45] Takacs, E., and Gillaspy, J. D. 2002. Am. Inst. Phys. Conf. Proc. 606: 151.

[46] Chen, G. X. et al. 2006. Phys. Rev. Lett. 97: 143201.

[47] Gillaspy, J. D. et al. 2011. Astrophys. J. 728: 132.
[48] Liang, G. Y. et al. 2009. Astrophys. J. 702: 838.

[49] DeWitt, D. R. et al. 1993. Phys. Rev. A 47: R1597.

[50] Nakamura, N., Kato, D., Niura, N., and Ohtani, S. 2000. J. Phys. Soc. Jpn. 69: 3228. 